\title{
A PREEMPTABLE SLOTTED ACCESS PROTOCOL TO TRANSPORT VARIABLE SIZE PACIKETS IN ALL-OPTICAL RING LANS/MANS
}

\author{
Marcos Rogério Salvador, Sonia Heemstra de Groot, Diptish Dey \\ Centre for Telematics and Information Technology, University of Twente; P.O. Box 217, 7500 \\ AE, Enschede, The Netherlands \\ \{salvador, heemstra, dey\}@cs.utwente.nl
}

\begin{abstract}
We describe a slotted access protocol that employs pre-emption to transport variable size packets. A node tries to transmit a packet using as many consecutive slots as possible. If a node detected a busy slot coming before it finished the transmission of a packet, that node will transmit until the current slot is full and will then stop. The node will resume transmission upon arrival of an empty slot.
\end{abstract}

Key words: $\quad$ MAC, Protocol, All-optical Networks, LAN, MAN

\section{INTRODUCTION}

A few medium access control (MAC) protocols that achieve all-optical packet switching in local area networks (LANs) or metropolitan area networks (MANs) have been reported in the literature. In this paper, we concentrate on MAC mechanisms for all-optical rings only.

The MAC protocols that have been proposed can be classified into either slotted ring with spatial reuse or token passing. Whilst each of these protocols has advantages and disadvantages compared to the others, none of them can transport variable size packets with both the efficiency and the flexibility desired. This is a considerable drawback in networks like the Internet, in which packet sizes are variable.

The protocol we describe in this paper is an attempt to cope with packet size distributions like that found in the Internet. Like most of the proposed 
protocols, our protocol is based on the slotted ring with spatial reuse concept. To transport variable size packets, the protocol additionally employs a pre-emption mechanism. The use of pre-emption allows for the transport of variable size packets while achieving the high performances and maintaining the simplicity of slotted ring with spatial reuse.

The rest of this paper is described as follows. In Section 2 we briefly introduce the protocols that have been proposed in the literature and show their weaknesses. In Section 3 we describe our protocol. In Section 4 we show and discuss some performance results obtained in simulation activities. In Section 5 we conclude the paper with some final thoughts.

\section{RELATED WORK}

The MAC protocols that have been proposed in the literature can be classified into two basic classes, namely, slotted ring with spatial reuse and token passing.

Examples of slotted ring with spatial reuse MAC protocols include [1,2, $3,4]$. In these protocols, the total capacity of the ring is divided into (time) slots of fixed length that rotate around the ring. Access to the medium is allowed only from the beginning of a slot and provided that that slot is empty. Slots are released at destination nodes and can be reused right away.

Despite the simplicity and high performance of slotted ring with spatial reuse, the fixed length of slots may lead to an inefficient transport of variable size packets. To overcome this limitation, the protocols described in $[1,2,3$, 4] work at packet aggregates rather than at individual packets. Upon arrival of an empty slot, as many packets destined to the same egress node as possible are transmitted. To avoid head-of-line (HOL) delays, packets are queued according to their destination nodes, which adds complexity to the protocols.

A token passing MAC protocol is described in [5]. In this protocol asynchronous access is controlled by multiple control tokens, each on a separate channel. Transmission of (one or more) variable size packets is allowed as long as the token is held. The token inter-arrival time determines the token holding time (THT) of a token based on the actual holding time of the previous token.

The distinct feature of this protocol is the fact that it considers the multiple channels as a single resource. Nevertheless, source removal of packets prevents the protocol from fully exploiting the network capacity. This is a considerable drawback in all-optical networks, where bandwidth * delay products are expected to be high. 


\section{MAC PROTOCOL}

We now describe a protocol that works at the packet level and achieves high performances. Very importantly, the protocol is not constrained to any particular node architecture in terms of transmission and reception capabilities. Rather, the protocol assumes the conceptual node architecture that is shown in Figure 1 and upon which most designs are based.

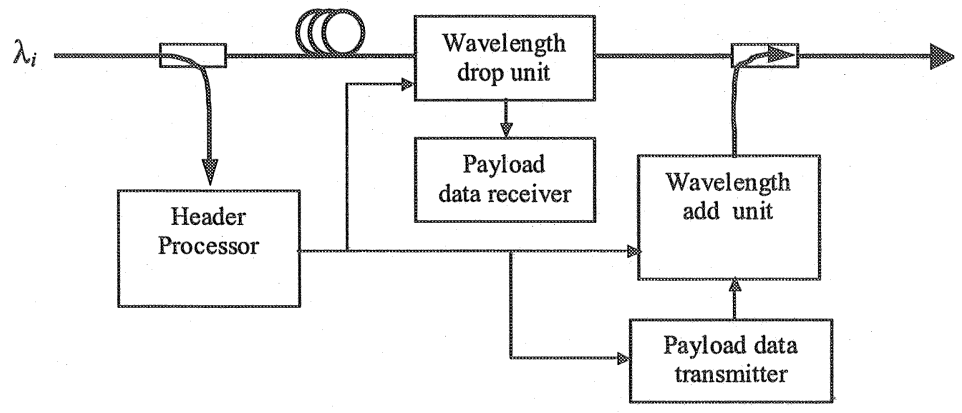

Figure 1. Conceptual node architecture

In this conceptual architecture, control information is somehow obtained, converted to the electronic domain and processed. Meanwhile, payload data information is held up in fibre delay lines (FDLs). When the payload data reaches the wavelength drop unit, the latter will have already been set-up by the header processor to either drop the slot or let the slot go. The same holds for the wavelength add unit. If the header processor detects an empty slot, then it signals the wavelength add unit to add into the slot's channel for the slot length and signals the payload data transmitter to start transmission.

The protocol is an adaptation of Carrier Sense Multiple Access/Ring Network (CSMA/RN) [6] to the slotted mode. CSMA/RN is an asynchronous network architecture that employs pre-emption and spatial reuse to achieve very high performances. The basic idea of CSMA/RN is that nodes can transmit as long as the medium is sensed idle. If a node is transmitting a packet and it detects traffic coming from upstream nodes, then that node stops transmitting the packet immediately. At the same time, that node sends a signal informing the destination node that the transmission has been interrupted and will be resumed later.

The major objective of our design is to achieve a transport capability that is nearly as efficient as CSMA/RN but without the complexity of CSMA/RN. CSMA/RN employs a tight synchronisation mechanism to cope with collisions. At the bit rates all-optical networks are meant and considering the packet size distribution of the Internet an even tighter 
synchronisation mechanism is required. The implementation of such a mechanism is not feasible with the state-of-the-art of all-optical networking technologies.

According to [7], packets in the Internet are variable and mostly small, with peaks at $40,44,552,576,1500$ bytes. More specifically, while approximately $60 \%$ of the packets are equal to or smaller than 44 bytes, approximately $50 \%$ of the traffic are carried in 1500 -byte packets.

Our protocol can transport such variable size packets using the very same synchronisation mechanism that is used in $[1,2,3,4]$. This is because upstream traffic on the ring can arrive at constant intervals only, regardless of the size of the traffic's packet.

The idea of the protocol is that the transmission of very small packets (e.g., 44 bytes) is never pre-empted by upstream traffic. To make sure that this happens, such a packet has to fit in a single slot. In other words, the slot size must not be smaller than 44 bytes, for instance.

The transmission of a packet that is larger than the slot size may span several slots. In this case, maximum efficiency is achieved when the packet can be transmitted using consecutive slots. Nevertheless, this can not be guaranteed. If a node that is transmitting such a packet detected the arrival of a busy slot, that node will fill the current slot completely and then will hold on its transmission. Upon arrival of an empty slot, that node will resume transmission. Figure 2 illustrates the basic idea of the protocol.

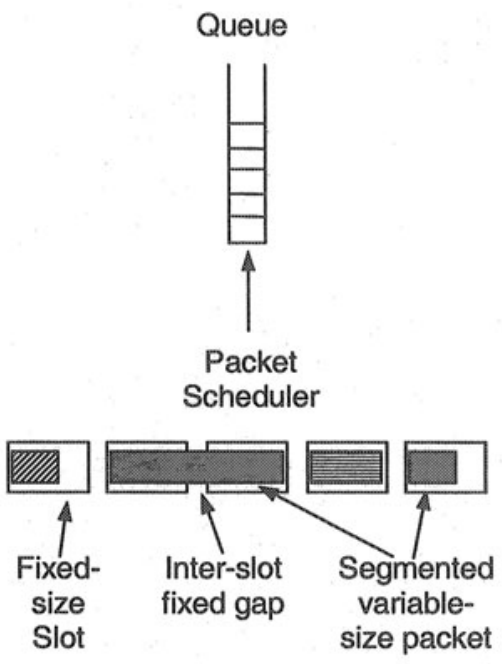

Figure 2. Illustration of the functioning of the protocol 
Since the slot size must not be too large for efficiency reasons (considering the packet sizes distribution in the Internet), a concern is the protocol processing bottleneck. Although protocol processing is getting faster each day, it is doubtful that network interface card (NIC) designs will keep up with the capacity enabled by technologies like wavelength division multiplexing (WDM) at reasonable cost and complexity.

To reduce processing demands, the protocol exploits the likelihood that correlated slots will travel together, i.e., one consecutively after the other. By correlated slots we mean slots carrying either an entire packet or fragments of a given packet.

The lighter the network load, the higher the number of empty slots circulating on the ring. Consequently, the higher the likelihood that correlated slots will travel together.

Correlated slots need not all to carry protocol control information (PCI) overhead. Only the first slot of a group of correlated slots, carrying either an entire packet or a fragment of a packet, which may not necessarily be the first one, needs to do it. This is because:

- traffic on the ring has priority over traffic awaiting for transmission;

- transmission of a new packet can only start after the packet selected previously has been completed transmitted.

In other words, PCI overhead suppression is possible because the protocol assures that fragments of a given packet arrive all in the order of transmission (or do not arrive at all as will be explained later on).

Our protocol attempts to reduce PCI overhead on both the control channel(s) and the payload data channel(s). PCI overhead on a control channel contains routing information basically. PCI overhead on a payload data channel contains the information that receivers use to re-assemble packets before sending them to the upper layer.

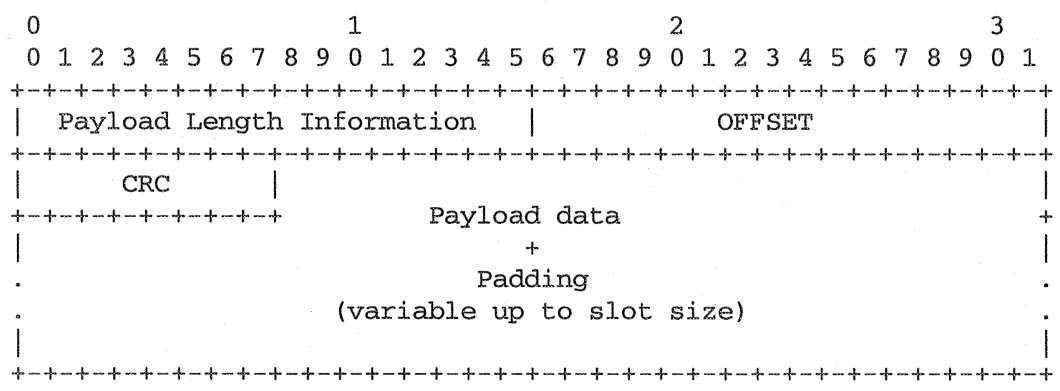

Figure 3. Payload frame layouts 
As far as payload data channels are concerned, only the first slot of a group of correlated slots carries PCI overhead. The consecutive slots carry no PCI information whatsoever. Figure 3 depicts the layout of the payload frame, which is defined using a 32-bit word alignment, as recommended by standardization organizations.

The fields defined in the payload frame are described as follows:

- Payload Length Information (PLI): 16-bit field that defines the size of a packet in bytes. PLI is set to all Os when carrying a fragment other than the first of a packet;

- OFFSET: 16-bit field that is used by destination nodes to re-assembly fragments back to original packets;

- CRC: 8-bit field that carries the 8-polynomial that is calculated over PLI and OFFSET;

- Payload data: variable size field that carries payload data or packets received from an upper layer;

- Padding: variable size field that is used to fill up a frame with 0s. In principle, the same PCI overhead suppression scheme adopted for payload slots could be used for control slots as well. However, it would be more difficult to detect malfunctioning and failures on the control channel(s) and to recover from them. Thus, we adopt a different scheme on the control channel(s): a reduced frame layout.

Figure $4 \mathrm{a}$ depicts the layout of the complete control frame. This frame layout is used in the following situations:

- A node starts transmitting a packet;

- A node resumes transmission immediately after it forwarded a busy slot. Figure $4 \mathrm{~b}$ depicts the layout of the reduced control frame (using a 32-bit word alignment). This frame layout is used in the following circumstances:

- A node is transmitting is situations other than those that define the use of the complete control frame; 


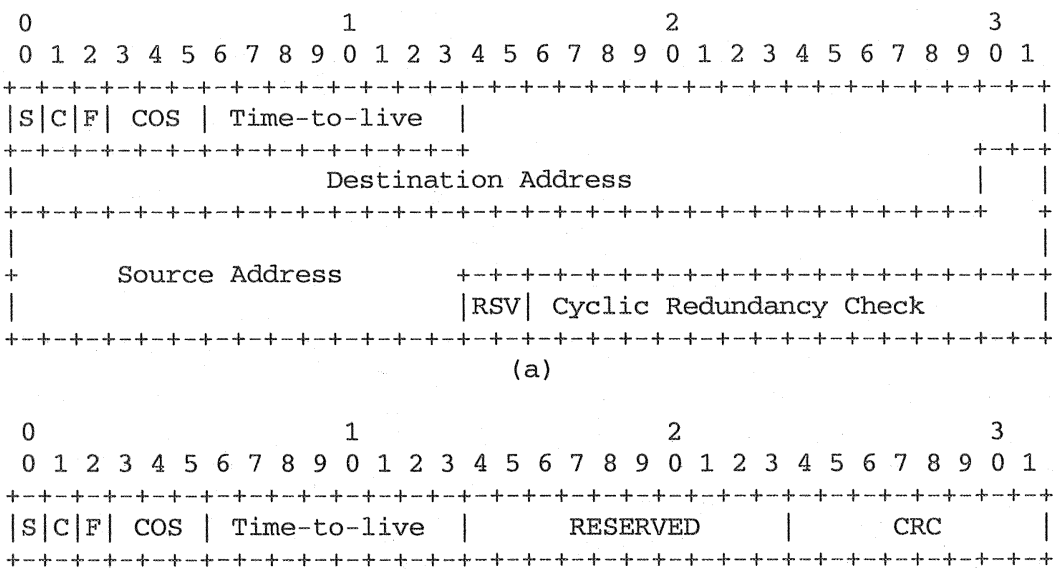

(b)

Figure 4. Header frame layouts

- The slot is empty.

The fields defined in both Figure $4 \mathrm{a}$ and Figure $4 \mathrm{~b}$ are defined as follows:

- S: 1-bit status field. The corresponding payload frame is empty if $S$ is set to 0 and busy otherwise;

- C: 1-bit field that indicates whether or not this slot immediately follows a correlated slot. $\mathrm{C}$ set to 0 indicates that the slot immediately follows a correlated one. $\mathrm{C}$ set to 1 indicates the opposite. $\mathrm{C}$ is processed only if $\mathrm{S}$ is set to 1 ;

- F: 1-bit fairness field. The header frame contains the fairness signal if $F$ is set to 1 and does not contain the fairness signal if otherwise;

- COS: 3-bit field used to indicate the class of service assigned to the data contained in the corresponding payload frame. COS can be useful in dealing with contention at destination nodes ${ }^{1}$ or cross-connect nodes. If $S$ is set to 0 , then COS is filled with 0 s and is not processed;

- Time-to-live: 8-bit field used to ensure that a payload frame does not circulate on the ring forever. This could be the case, for instance, if both the destination node and the source node failed while a slot is on the ring. Time-to-live is decremented at each node. A header frame and its corresponding payload frame are forwarded only if Time-to-live is greater than 0 ; otherwise they are both silently discarded. If $\mathrm{S}$ is set to 0 , then Time-to-live is filled with 0s and is not processed;

${ }^{1}$ Destination contention may occur at a node equipped with a number of tuneable receivers that is smaller than the number of channels in the network. 
- Destination Address (DA): 48-bit IEEE standard MAC address used to determine whether a payload frame is or is not destined to the node processing it. IEEE MAC address is used for interoperability purposes;

- Source Address (SA): 48-bit IEEE standard MAC address. SA is used either to perform source release of multicast/broadcast payload frames or to ensure that unicast payload frames are released should destination release fail;

- RESERVED: 30-bit field reserved for possible use in the future;

- CRC: 16-bit field that carries the cyclic redundancy check 16-polynomial calculated over all the other fields.

The protocol follows the end-to-end argument [8] and is unreliable. No error correction mechanism of any kind is used in the protocol whatsoever. Only error detection over PCI overhead is employed. Error detection over payload data is left to the upper layers. There are two reasons for this. First, transmission errors are very seldom. Second, most errors occurs at NICs. Thus, performing error detection over payload data at the MAC layer may be useless as errors might occur when transferring the data to the upper layer.

The protocol responds to the detection of an error in two distinct ways depending on whether that error occurred on a control frame or on a payload frame:

- Control frame: the control frame and its corresponding payload frame are discarded. In other words, the corresponding payload frame is freed for reuse. The same behaviour applies to the subsequent correlated slots in the group;

- Payload frame: payload data contained in the frame and all the correlated payload data that have been buffered are discarded. All next incoming payload frames coming from the same node and whose PLI field is filled with 0 s are discarded as well.

Due to destination release, under certain traffic conditions some nodes may get better-than-average access to the medium while others may get worse-than-average access to the medium. The protocol uses the fairness mechanism proposed in [9] to correct this behaviour.

The fairness mechanism works as follows. Upon two visits of the socalled SAT signal, a node is allowed to transmit up $\mathrm{k} \geq 1>0$ data units. If upon visit of the SAT signal a node either has transmitted at least 1 data units or has no packets waiting for transmission, the node's quota is renewed and the SAT signal is immediately forwarded to the next node. Otherwise, the node holds the SAT signal until it has transmitted at least 1 data units. The node's quota is then renewed and the SAT signal is forwarded to the next node.

Transmission quota is expressed in slots in our protocol. This is because a busy payload slot, even if carrying a single byte of data, can not be reused 
(i.e., the remaining space available) by any node until it is released. Thus, there is no point in expressing quota in some other unit, e.g., bits, as doing this could lead to a situation in which a node is prevented from completely filling a slot simply because its quota exhausted.

In our protocol, fairness is enforced on a channel basis. This is to prevent nodes transmitting on highly loaded channels from affecting nodes transmitting on lightly loaded channels. If only a single signal is used in the network, nodes transmitting on highly loaded channels will hold the SAT signal and nodes transmitting on lightly loaded channels will be prevented from transmitting while the SAT signal does not arrive.

\section{PERFORMANCE ANALYSIS}

We now shall look at the performance characteristics of the protocol. Due to the difficulties in modelling slotted ring networks with spatial reuse analytically, we focus our discussions on the data that was obtained in an object-oriented, event-driven simulator that has been developed using Java.

To assess how the protocol deals with concurrent access to the ring, we assume a $20 \mathrm{~km}$ ring in which $\mathrm{N}=4$ nodes share a given channel. The bit rate of the channel is $2.5 \mathrm{Gbps}$. We consider slots with two payload data lengths: 64 bytes and 128 bytes. Therefore, two ring lengths in terms of number of slots are considered: 431 slots and 223 slots. Values 1 and $\mathrm{k}$ of the fairness mechanism are set both to the number of slots in the network.

Packets are generated according to a Poisson process with fixed rate $\lambda$ and destinations equally likely. All nodes generate the same amount of traffic. The offered load in the network is therefore the sum of all the traffic generated at each node.

Table $I$ shows the packet size distribution used in the simulations. This distribution is based on the packet size distribution found in wide area networks (WANs) [7]. The values in Table 1 are based on such a distribution. 20 bytes are added to each size to reflect the most apparent effects of Internet Protocol (IP) version 6 traffic.

Table 1. Packet sizes distribution

\begin{tabular}{ll}
\hline Packet size (bytes) & Percentage of packets \\
\hline 64 & 60 \\
596 & 15 \\
700 & 5 \\
800 & 5 \\
1100 & 7 \\
1500 & 8 \\
\hline
\end{tabular}


Figure 5 shows the network throughput as a function of the offered load. Network throughput is defined as the sum of the throughput of each node. Node throughput is calculated over the total number of bits of payload data transmitted divided by the channel capacity available during the simulation period.

Two are the conclusions from Figure 5. First, below the saturation point the network throughput is nearly twice as much the network capacity. This is due to destination release and symmetric traffic distribution. Second, the network achieves higher throughputs when the 64-byte packets fit exactly in the slots. This is due to the fact that approximately $60 \%$ of all the packets generated are transmitted with minimum overhead. Albeit not shown, the bigger slot size carries approximately four times as much PCI overhead as the smaller slot size.

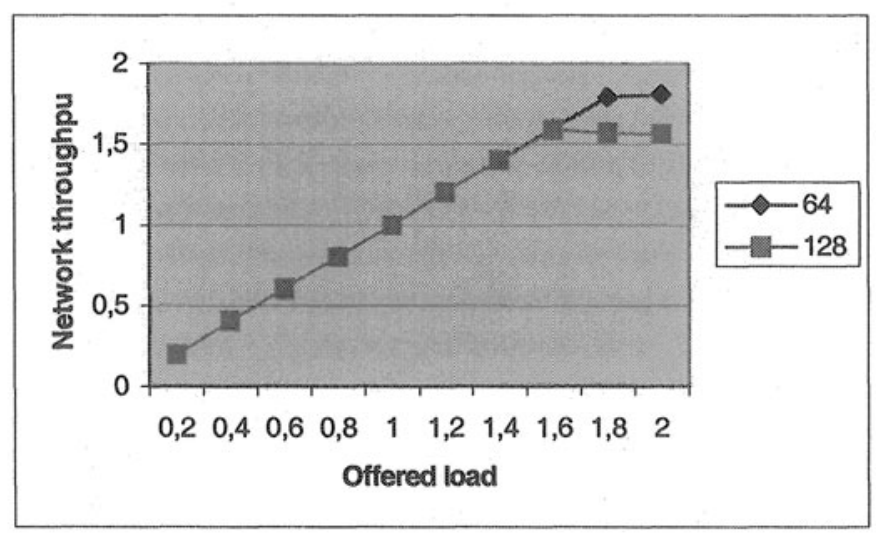

Figure 5. Network throughput

Before we continue, it should be noted that from now on all the measures concern nodes rather than the network. However, due to the fairness mechanism that ensures fair access to the ring, the nodes all show equal results. Therefore, for the sake of clearness we show only one curve per slot size.

Figure 6 shows the packet loss ratio. Packet loss ratio is defined as the number of discarded packets divided by the number of received packets. The queue size is assumed to store up to $64 \mathrm{Kbytes}$, as used in many commercial products. Other queue sizes may yield different results. 


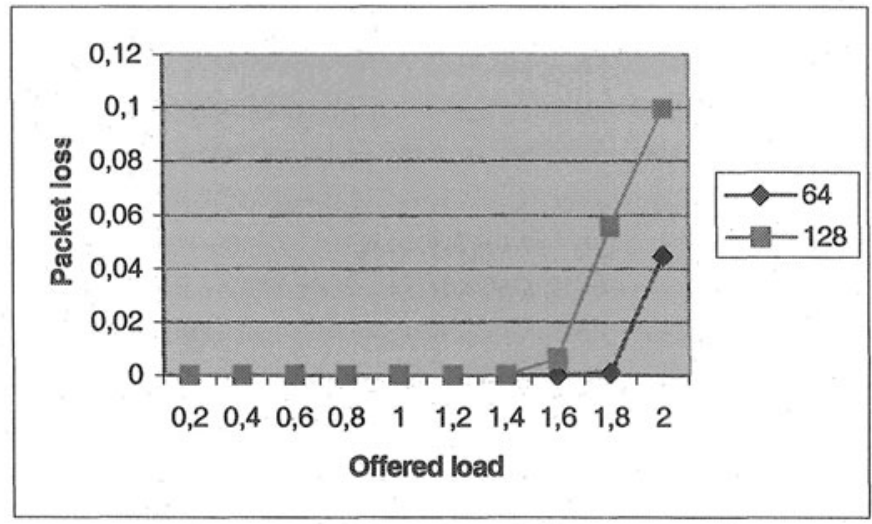

Figure 6. Packet loss

The curves show that the network can sustain network loads much higher than its nominal capacity. With 128-byte slots, loads of up to approximately 1.45 times the network capacity are sustained without discarding a single packet. With 64-byte slots, this figure goes to approximately 1.7. This difference stems from the fact that 128-byte slots carry more overhead (i.e. padding) than 64-byte slots.

Figure 7 shows node average access delay. Access delay is defined as the time gap between the transmission of the last bit of packet $p$ and the transmission of the last bit of packet $p-1$. Average access delay is the sum of all the access delays divided by the number of transmitted packets.

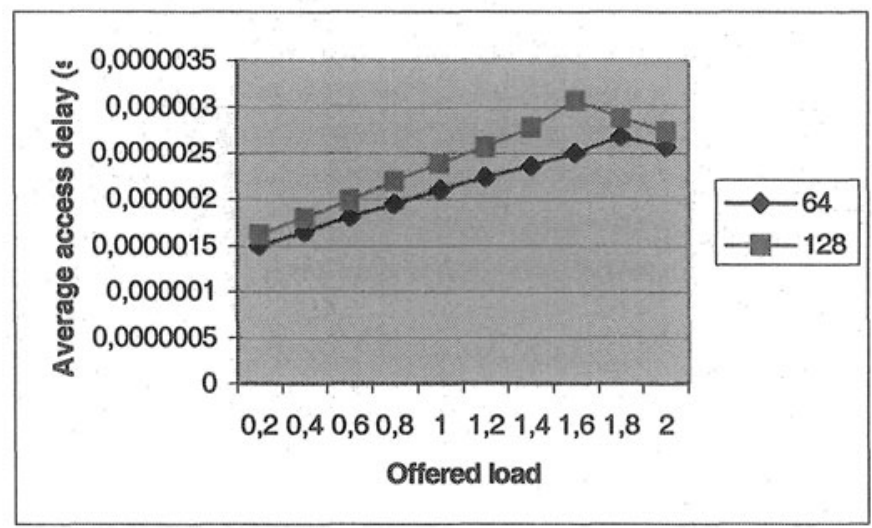

Figure 7. Average access delay 
Two conclusions can be drawn from Figure 7. First, average access delay is higher when using 128-byte slots. Nevertheless, the difference is not proportional to the difference in size between the two slot sizes. This is because of the gains in transmitting big packets in 128-byte slots. Second, average access delay increases smoothly regardless of the slot size. This is due to the fact that even at high loads, there is still a considerable number of (non-contiguous) empty slots circulating in the ring that help to keep access delays stable.

The decay of the curves above their peak is due to congestions and, consequently, packet discarding.

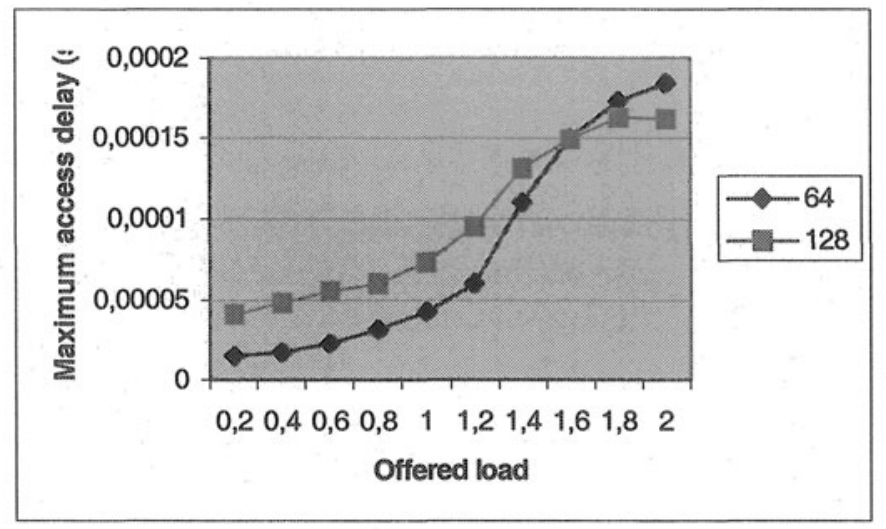

Figure 8. Maximum access delay

Figure 8 shows the maximum access delays (out of 10 simulation runs) experienced in the network. As expected, maximum access delay is higher with 128-byte slots. This is due to the fact that the number of slots circulating on the ring is approximately half compared to 64-byte slots.

The curves show that maximum access delay increases slightly with offered loads below 0.8 regardless of the slot size. This stems from the fact that at this load and below, approximately half of the slots circulate empty.

As the network utilisation reaches approximately $70 \%$ of its maximum, maximum access delay increases more sharply. At this point, there are not to many empty slots circulating in the ring and those that are empty are likely not consecutive. These together increase packet fragmentation and access delays.

With the network utilisation getting close to its maximum, congestion starts at the nodes. As nodes discard packets, the maximum access delay curves tend to get smoother. 


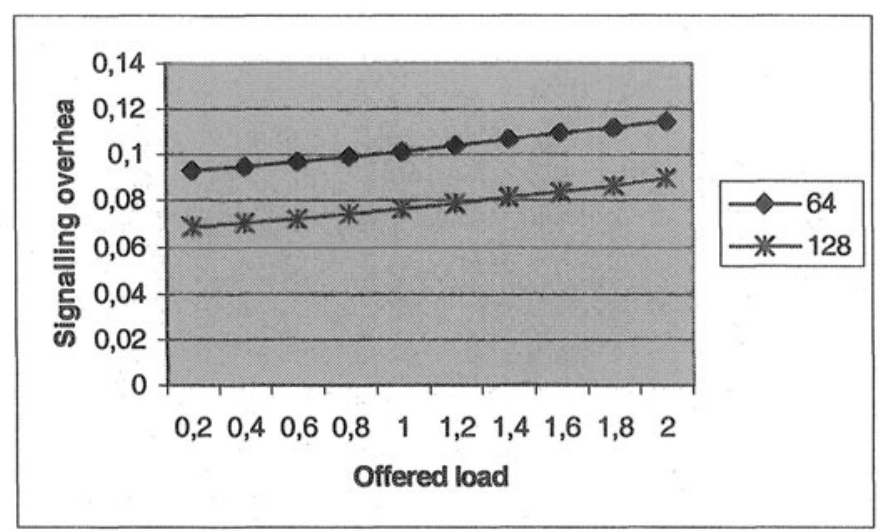

Figure 9. Signalling overhead

Figure 9 shows average per node signalling overhead. Signalling overhead is the number of bits of signalling transmitted divided by the number of bits of payload data transmitted. This explains why the two curves are alike.

The fact that the curves increase smoothly shows that the gains that can be obtained by transmitting using consecutive slots become more apparent at higher loads. The fairness mechanism comes into play in the sense that a node holds the SAT to back-off other nodes. Consequently, that node gets access to a higher number of consecutive empty slots, which in turn, leads to higher efficiency.

Figure 10 shows per node signalling channel consumption as a function of network throughput. Signalling channel consumption is the number of bits of signalling transmitted by a node divided by the bandwidth available during the simulation time. 


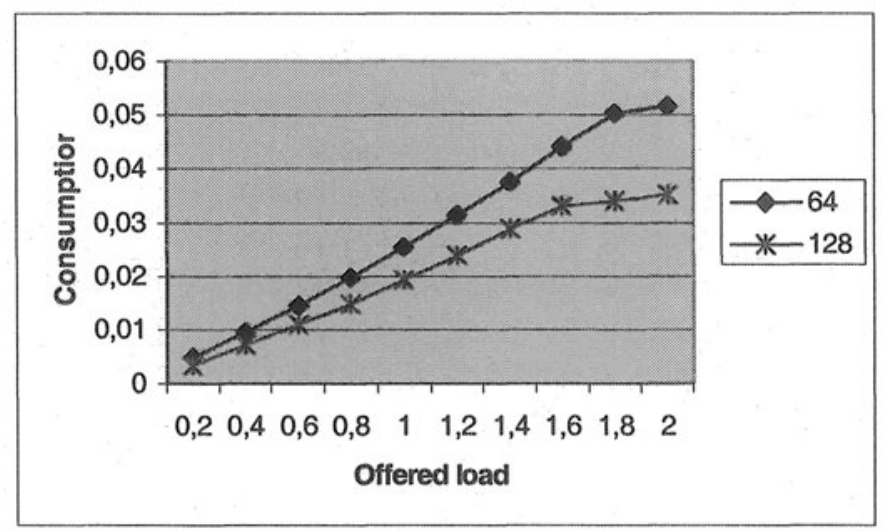

Figure 10. Signalling channel consumption

As expected, 128-byte slots lead to lower signalling demands. However, the gains in consumption are not proportional to the difference in size between the two slot sizes. This is due to the fact that much of the gain is eliminated by the need to transmit 64-byte packets.

The two curves show that signalling channel consumption worsens more rapidly when using 64-byte slots. This is because as the network load increases, more slots are required to cope with the load. Therefore, as the network load increases it becomes more apparent the inefficiency of 64-byte slots in carrying packets bigger than 64 bytes.

\section{CONCLUSIONS}

We described a MAC protocol that transports variable size packets in alloptical packet-switched LANs and MANs. Very importantly, the protocol achieves that without the need for complex mechanisms. As a matter of fact, it is slightly more complex than the slotted ring with spatial reuse protocols that have been proposed in $[1,2,3,4]$.

Another important feature of the protocol is that can work in multichannel network and node architectures that have been proposed in the literature.

A drawback of the protocol is the need for receiving buffers in network interface cards (NICs). Each NIC has to maintain one buffer per possible sender, i.e., N-1 buffers. Nevertheless, due to the fact that the protocol is unreliable, each buffer must be not larger than a maximum transmission unit (MTU)-sized packet. 


\section{ACKNOWLEDGMENTS}

This research is part of project Flamingo and is supported by the Dutch Technology Foundation STW, applied science division of NWO and the technology programme of the Ministry of Economic Affairs of The Netherlands.

\section{REFERENCES}

[1] M. A. Marsan, et al. All-Optical WDM Multi-Rings with Differentiated QoS. In IEEE Communications Magazine, pp. 58-66, February 1999.

[2] M. R. Salvador, S. Heemstra de Groot, and D. Dey. An All-Optical WDM PacketSwitched Network Architecture with Support for Group Communication. In Procs. of IEEE International Conference on Networking (ICN), LNCS 2093, pp. 326-335, Colmar, France, July 9-13, 2001.

[3] 1. Chlamtac, et al. Scalable WDM Access Network Architecture Based on Photonic Slot Routing. In IEEE/ACM Transactions on Networking, Vol. 7, No. 1, February 1997.

[4] M. A. Marsan et al.. MAC Protocols and Fairness Control in WDM Multi-Rings with Tunable Transmitters and Fixed Receivers. In IEEE/OSA Journal of Lightwave Technologies, Vol. 14, No. 6, June 1996.

[5] J. Cai, A. Fumagalli, and I. Chlamtac. The Multitoken Interarrival Time (MTIT) Access Protocol for Supporting Variable Size Packets Over WDM Ring Network. In IEEE Journal of Selected Areas in Communications, pp. 2094-2104, Vol. 18, No. 10, October 2000.

[6] E. C. Foudriat, K. Maly, C. M. Overstreet, S. Khanna, and F. Paterra. A Carrier Sensed Multiple Access Protocol for High Data Rate Ring Networks. ACM Computer Communications Review, Vol. 21, No. 2, April 1991, pp. 59-70.

[7] K. Claffy, G. Miller, and K. Thompson. The nature of the beast: recent traffic measurements from an Internet backbone. In Procs. of Internet Society (ISOC) Internet Summit (Inet), Geneva, Switzerland, 21-24 July, 1998.

[8] J. H. Saltzer, D. P. Reed, and D. D. Clark. End-to-End Arguments in System Design. In ACM Transactions in Computer Systems, Vol. 2, No. 4, pp. 277-288, November 1984.

[9] Y. Ofek. Overview of the Metaring architecture. In Computer Networks and ISDN Systems, 26(6-8), 817-8 